\title{
Fingolimod (FTY-720) is Capable of Reversing Tumor Necrosis Factor Induced Decreases in Cochlear Blood Flow
}

\author{
*Mattis Bertlich, *Friedrich Ihler, *Bernhard G. Weiss, †łSaskia Freytag, *Mark Jakob, \\ $\S$ Michael Strupp, ||Hannah Pellkofer, and *Martin Canis
}

\begin{abstract}
* Department of Otorhinolaryngology, Head and Neck Surgery; †Population Health and Immunity Division, Walter and Eliza Hall Institute; †Department of Medical Biology, University of Melbourne, Parkville, Australia; §Department of Neurology and German Center for Vertigo and Balance Disorders, Munich University Hospital, Munich; and ||Department of Neurology, University Medical Center Göttingen, Göttingen, Germany
\end{abstract}

\begin{abstract}
Hypothesis: The potential of Fingolimod (FTY-720), a sphingosine-1-phosphate analogue, to revoke the changes in cochlear blood flow induced by tumor necrosis factor (TNF) was investigated.

Background: Impairment of cochlear blood flow has often been considered as the common final pathway of various inner ear pathologies. TNF, an ubiquitous cytokine, plays a major role in these pathologies, reducing cochlear blood flow via sphingosine-1-phosphate-signaling.

Methods: Fifteen Dunkin-Hartley guinea pigs were randomly assigned to one of three groups (placebo/placebo, TNF/ placebo, TNF/FTY-720). Cochlear microcirculation was quantified over 60 minutes by in vivo fluorescence microscopy before and after topical application of placebo or TNF
\end{abstract}

$(5 \mathrm{ng} / \mathrm{ml})$ and after subsequent application of placebo or FTY-720 $(200 \mu \mathrm{g} / \mathrm{ml})$.

Results: Treatment with TNF led to a significant decrease of cochlear blood flow.

Following this, application of placebo caused no significant changes while application of FTY-720 caused a significant rise in cochlear blood flow.

Conclusions: FTY-720 is capable of reversing changes in cochlear blood flow induced by application of TNF. This makes FTY-720 a valid candidate for potential treatment of numerous inner ear pathologies. Key Words: Cochlear blood flow-Fingolimod-Microcirculation-Tumor necrosis factor.

Otol Neurotol 38:1213-1216, 2017.
Local microcirculation is a critical parameter for cochlear function. Impairment of microcirculation results in decreases in partial oxygen pressure (1) and subsequent loss of function, including significant decreases in endocochlear potential and increases in hearing thresholds (2-4). Impairment and regulation of cochlear microcirculation also plays a role in the

Address correspondence and reprint requests to Mattis Bertlich, M.D., Department of Otorhinolaryngology, Head and Neck Surgery, University Medical Center Göttingen, Robert-Koch-Strasse 40, 37075 Göttingen, Germany; E-mail: Mattis.Bertlich@med.uni-goettingen.de.

H.P. and M.C. have contributed equally to this study.

This study was supported by a grant by the Deutsche Forschungsgemeinschaft to Professor Canis under the grant code CA629/2-1.

The authors M.B., F.I., B.G.W., S.F., M.J., M.C. declare that they have no conflicts of interest. M.S. is Joint Chief Editor of the Journal of Neurology, Editor in Chief of Frontiers of Neuro-otology and Section Editor of F1000. He has received speaker's honoraria from Abbott, Actelion, Biogen, Eisai, GSK, Henning Pharma, Interacoustics, MSD, Otometrics, Pierre-Fabre, TEVA, UCB. He acts as a consultant for Abbott, Actelion, IntraBio, and Sensorion. H.P. has received travel grants from Bayer and Novartis and speaker's honoraria from Bayer, Biogen, Teva.

Supplemental digital content is available in the text.

DOI: $10.1097 /$ MAO.0000000000001510 pathophysiology and treatment of other pathologies such as Menière's disease (5) and inflammatory inner ear pathologies $(3,6)$.

Tumor necrosis factor (TNF) is an ubiquitous cytokine that is involved in various types of disease, including, but not limited to malignancies (7), autoimmune diseases (8), and depression (9). It has also been established that TNF is capable of impairing local circulation in various tissues, including the cochlea $(6,10)$. Moreover, several studies have been able to demonstrate that TNF is involved in a number of inner ear pathologies, such as sudden sensorineural hearing loss (6), acoustic (3), and physical trauma (11).

Research has shown that the effects of TNF are mediated by several pathways (12): after binding to its specific receptor, TNF amplifies intracellular signaling pathways that lead to induction of cell death, including NF BB- and JNK-signaling as well as caspases. TNF also activates sphingosine-1-phosphate signaling (6). Overall, the short-term effects of TNF related pathologies are mediated by sphingosine-1-phosphate signaling (6) while the long-term effects are instead mediated by intracellular signaling leading to cell death, including NFкB- and JNK-signaling (13). 
All substances that have been used to reverse shortterm effects of TNF on cochlear microcirculation have either not been specific to sphingosine-1-phosphate signaling (14) or have been experimental and have not been approved for human use (6). However, since 2010, Fingolimod (FTY-720), a sphingosine-1-phosphate analogue that is sold under the name Fingolimod has been approved for clinical use in human patients suffering from multiple sclerosis $(15,16)$. Upon binding the sphingosine-1-phosphate receptor, it is actively transported into the expressing cell and thus cannot be activated anymore $(17,18)$. Hence, we examined the potential of FTY-720 to reverse changes in cochlear microcirculation induced by topical application of TNF.

\section{MATERIALS AND METHODS}

\section{Ethics Statement}

All experiments were performed according to local state regulations and approved by the responsible authorities (Niedersächsisches Landesamt für Verbraucherschutz und Lebensmittelsicherheit LAVES) under the animal license no: 33.942502-04-14/1427.

\section{Animals}

Animals were Dunkin-Hartley guinea pigs bred for experimental use and purchased from Harlan Laboratories (OberRamstadt, Hesse, Germany), weighing 200 to $450 \mathrm{~g}$. Anesthesia was induced by an intraperitoneal injection of $50 \mathrm{mg} / \mathrm{kg}$ bodyweight (b.w.) ketamine and $5 \mathrm{mg} / \mathrm{kg}$ b.w. xylazine and sustained by repeated intramuscular injections of $25 \mathrm{mg} / \mathrm{kg}$ b.w. ketamine and $2.5 \mathrm{mg} / \mathrm{kg}$ b.w. xylazine every 30 minutes.

Surgical preparation lasted approximately 60 to 90 minutes. After the experiments were conducted, animals were euthanized by an overdose of anesthesia and subsequent cervical dislocation.

\section{Surgical Approach}

The technique applied in this experiment has been previously described (19-21). After induction of anesthesia, a cervical venous catheter was surgically implemented. Subsequently, the external ear and the temporal bone covering the bulla were removed, thus exposing the cochlea. Overlying periosteal vessels were carefully removed using a microsponge. As soon as all the vessels were removed, a rectangular window was carved into the cochlea above the second turn, exposing the stria vascularis. Finally, fluorescein-labeled dextrane (molecular weight 500,$000 ; 0.05-0.1 \mathrm{ml}$ of a $5 \%$ solution in $0.9 \% \mathrm{NaCl}$; Sigma-Aldrich, Deisenhofen, Germany) was applied intravenously, allowing direct visualization of intravascular blood flow in the stria vascularis, with a contrast between erythrocytes and plasma. Following illumination with a Leica EL6000 light source (Leica Microsystems, Wetzlar, Germany), images were obtained with a Leica M205 FA stereomicroscope. The proprietary Leica Application Suite software was then used to process and save the generated data for later off-line analysis. An example of the video material acquired is available with the supplementary digital content (http://links.lww.com/ $\mathrm{MAO} / \mathrm{A} 538)$.

Velocity (micrometers per second) and diameter (micrometers) of stria vascularis vessels were quantified using CapImage (Dr. Zeintl Biomedical Engineering, Heidelberg, Germany), a software specifically designed for this purpose
(22). An exact description with sample pictures of the digital quantification of intravascular blood velocity is also available with the supplementary digital content (http://links.lww.com/ $\mathrm{MAO} / \mathrm{A} 539)$.

For each vessel the intravascular blood flow was calculated with a formula proposed by Wayland: $q=(v / 1.6) \times(d / 2)^{2} \times \pi$ (23). To correct for interindividual differences as well as differences between individual vessels, units are reported as arbitrary units (AU), representing change from the initially acquired basal values.

\section{Treatment Protocol}

Five animals were randomly assigned to one of three groups (placebo/placebo, TNF/placebo, or TNF/FTY-720). After the surgical preparation and before treatment had begun, basal images were acquired for later analysis. Following this, placebo or TNF was applied topically for 20 minutes. Afterwards, the bulla was rinsed with $0.9 \%$ saline solution for 10 minutes and the microcirculation was quantified again. Then, placebo or FTY-720 was applied for 20 minutes and the bulla was washed again before final images were acquired. Similar protocols have been used in previous experiments addressing this topic $(10,14)$. After the protocol had been finished, the animals were euthanized.

For placebo, a sterile saline solution was chosen. The concentration of TNF was $5.0 \mathrm{ng} / \mathrm{ml}$ in sterile saline solution as reported in previous experiments $(10,14)$. For the FTY-720 solution, $1 \mathrm{mg}$ of FTY-720 was dissolved in $5 \mathrm{ml}$ of dimethyl sulfoxide, resulting in a concentration of $200 \mu \mathrm{g} / \mathrm{ml}$. This was then diluted 1:10 in sterile saline solution, resulting in a final concentration of $20 \mu \mathrm{g} / \mathrm{ml}$ FTY-720 which was then applied.

\section{Statistics}

Statistical analysis was carried out using Project R (Build 3.3.2 for Windows, The R Project for Statistical Computing, http://www.r-project.org/). To detect differences between the groups as well as between the timepoints within the groups, we used a Wilcoxon test. This test allows paired and unpaired twosample nonparametric comparisons. We used a Bonferroni correction to adjust for multiple testing, thus a $p$ value of $\alpha$ $<0.005$ was considered to be statistically significant.

\section{RESULTS}

\section{Effect of Placebo + Placebo on Cochlear Blood Flow}

The initial topical application of placebo leads to no significant changes in cochlear blood flow, which remained steady at $1.01 \pm 0.06 \mathrm{AU}$ (Fig. 1, left column). Subsequent application of placebo leads to a drop in cochlear blood flow to $0.92 \pm 0.09 \mathrm{AU}$. There were no significant differences within the group (Table 1). The values acquired after the first treatment with placebo were significantly different from those values obtained after treatment with TNF (Table 2).

\section{Effect of TNF + Placebo on Cochlear Blood Flow}

Initial application of TNF led to a drop in cochlear blood flow to $.81 \pm .07 \mathrm{AU}$ (Fig. 1, middle column). This was significantly different from the basal values as well as compared with placebo (Tables 1 and 2). Subsequent application of placebo induced no change in cochlear blood flow, which remained steady at $0.81 \pm 0.10 \mathrm{AU}$. 


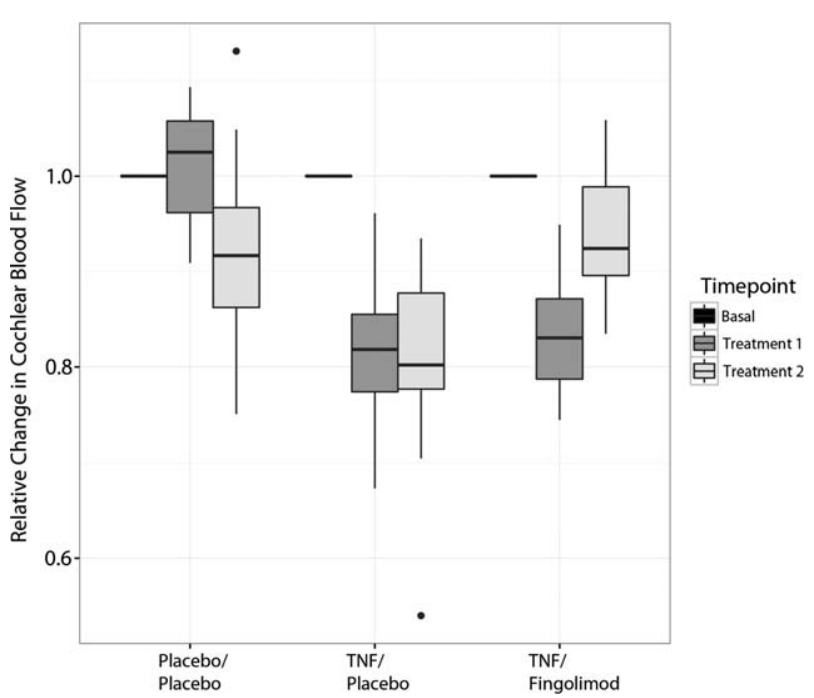

FIG. 1. Relative changes in cochlear blood flow over time, before initial treatment, in between treatments and after final treatment, reported in arbitrary units (AU).

This value was significantly different from the initial acquired basal value as well as the final values measured after application of TNF and subsequently FTY-720, but not significantly different from the values obtained within the same group after initial TNF application (Tables 1 and 2).

\section{Effect of TNF + FTY-720 on Cochlear Blood Flow}

Again, initial application of TNF caused a significant drop in cochlear blood flow to $0.83 \pm 0.06$ AU (Fig. 1, right column; Tables 1 and 2). Subsequent application of FTY-720 caused an increase in cochlear blood flow to $0.94 \pm 0.07 \mathrm{AU}$. This increase was significantly different from the previously obtained values within this group (Table 1) as well as significantly different from the endpoint of the group that was treated with TNF and subsequently placebo, but not different from the values that were obtained in the group after the twofold application of placebo (Table 2).

\section{DISCUSSION}

In this study we confirmed that TNF decreases cochlear blood flow after topical application. This finding is in

TABLE 1. $p$ Values for the comparisons of time points in between the groups. Values marked bold were considered significant

\begin{tabular}{lccc}
\hline & $\begin{array}{c}\text { Basal } \\
\text { Versus } \\
\text { Treatment } 1\end{array}$ & $\begin{array}{c}\text { Basal } \\
\text { Versus } \\
\text { Treatment 2 }\end{array}$ & $\begin{array}{c}\text { Treatment 1 } \\
\text { Versus } \\
\text { Treatment 2 }\end{array}$ \\
\hline Placebo/placebo & $p=0.561$ & $p=0.010$ & $p=0.005$ \\
TNF/placebo & $p<\mathbf{0 . 0 0 1}$ & $p<\mathbf{0 . 0 0 1}$ & $p=1.000$ \\
TNF/FTY-720 & $p<\mathbf{0 . 0 0 1}$ & $p=0.013$ & $p<\mathbf{0 . 0 0 1}$ \\
\hline
\end{tabular}

FTY-720 indicates Fingolimod; TNF, tumor necrosis factor.
TABLE 2. $p$ Values for the comparions of groups within time points. Values marked bold were considered significant

\begin{tabular}{lll}
\hline & Treatment 1 & Treatment 2 \\
\hline Placebo/placebo versus TNF/placebo & & $p=0.005$ \\
TNF/placebo versus TNF/FTY-720 & $p<\mathbf{0 . 0 0 1}^{a}$ & $p<\mathbf{0 . 0 0 1}$ \\
Placebo/placebo versus TNF/FTY-720 & & $p=0.539$ \\
\hline
\end{tabular}

${ }^{a}$ The animals of the TNF/placebo and the TNF/FTY-720 group at Treatment 1 were pooled and compared against the animals that had received placebo, since the treatment both groups had received was biologically the same.

FTY-720 indicates Fingolimod; TNF, tumor necrosis factor.

line with previous reports (6). Moreover, it has been reported that the effect of TNF is dose-dependent (14). It was previously also demonstrated that the effects of TNF on cochlear blood flow could be reversed by blocking the TNF receptor with etanercept $(6,14)$. Etanercept has, so far, been shown to have beneficial effects in various cochlear pathologies that coincide with impairments of cochlear blood flow, including sudden sensorineural hearing loss (6) or acoustic trauma (3).

We demonstrated that topical application of FTY-720 is able to reverse the effects of TNF on cochlear blood flow: application of FTY-720 caused a significant increase in cochlear blood flow. The fact that the cochlear blood flow had also decreased somewhat in the group that had received a placebo twice could be explained by increased clotting in the capillaries caused by the surgical manipulation, a phenomenon that has previously been reported in this animal model (20). Even though this could be a potential confounder in the animal model used in this study, this also raises the threshold levels to obtain significant results, assuming that FTY-720 would increase cochlear blood flow compared with previous values.

The effect of FTY-720 is most likely mediated by its interaction with the sphingosine-1-phosphate-receptor. It has been known to bind the receptor, causing it to be transported into the inside of the cell, thus preventing it from being activated (17). This viewpoint is supported by the fact that blocking of the sphingosine-1-phosphate receptor has been able to revoke typical effects of TNF on microvasculature $(6,10,24,25)$. Additionally, another potential mode of action has to be taken into account: FTY-720 has also been known to interact with the cannabinoid CB1-receptor; more specifically as a competitive CB-1-antagonist (26). It has been reported that antagonism at the CB-1-receptor is capable of preventing decreases in microcirculation caused by endotoxemia in other tissues, specifically the small intestine (27). This argument is further supported by the fact that endotoxemia is thought to cause several complications involving the TNF pathway and that blockage of the TNF-receptor using etanercept significantly reduces the immediate clinical effects of endotoxemia $(28,29)$.

The presented results show that FTY-720 is capable of reversing the effects of TNF on cochlear blood flow. Assuming that alterations in cochlear blood flow cause a 
drop in partial oxygen pressure (1) and thus eventually ischemia and subsequent apoptosis of inner hair cells, FTY-720 might be a promising agent fit for clinical testing in various inner ear pathologies, including sudden sensorineural hearing loss $(4,6)$, noise trauma (3), as well as physical trauma to the cochlea (11). As an additional benefit in comparison to other drugs that have been suggested as treatments for these pathologies, such as AM-111 (30) or JTE-013 (6), FTY-720 has already been approved for clinical use in relapsing remitting multiple sclerosis and, thus, has a well known safety profile. Moreover, its route of administration (oral intake, once daily) is significantly less complicated than the route of administration of etanercept, which has also been suggested as a potential treatment for these pathologies $(3,6,14)$ and which has to be applied by daily subcutaneous injections.

Common side-effects of FTY-720 include leukopenia with the potential risk of opportunistic infections, bradycardia, elevation of liver enzymes, arterial hypertension, and macular edema (31). Since the need of an observational period of 6 hours after the first intake and the need of regular follow up examinations including frequent control of liver enzymes and lymphocytes during therapy with FTY-720, the intratympanic installation and subsequent crossing into the cochlea might be considered.

\section{REFERENCES}

1. Lamm K, Arnold W. The effect of blood flow promoting drugs on cochlear blood flow, perilymphatic $\mathrm{pO}(2)$ and auditory function in the normal and noise-damaged hypoxic and ischemic guinea pig inner ear. Hear Res 2000;141:199-219.

2. Arpornchayanon W, Canis M, Suckfuell M, Ihler F, Olzowy B, Strieth S. Modeling the measurements of cochlear microcirculation and hearing function after loud noise. Otolaryngol Head Neck Surg 2011;145:463-9.

3. Arpornchayanon W, Canis M, Ihler F, Settevendemie C, Strieth S. TNF-alpha inhibition using etanercept prevents noise-induced hearing loss by improvement of cochlear blood flow in vivo. Int J Audiol 2013;52:545-52.

4. Ihler F, Strieth S, Pieri N, Gohring P, Canis M. Acute hyperfibrinogenemia impairs cochlear blood flow and hearing function in guinea pigs in vivo. Int $J$ Audiol 2012;51:210-5.

5. Nakai Y, Masutani H, Moriguchi M, Matsunaga K, Kato A, Maeda H. Microvasculature of normal and hydropic labyrinth. Scanning Microsc 1992;6:1094-7.

6. Scherer EQ, Yang J, Canis M, et al. Tumor necrosis factor-alpha enhances microvascular tone and reduces blood flow in the cochlea via enhanced sphingosine-1-phosphate signaling. Stroke 2010;41: 2618-24.

7. Lebrec H, Ponce R, Preston BD, Iles J, Born TL, Hooper M. Tumor necrosis factor, tumor necrosis factor inhibition, and cancer risk. Curr Med Res Opin 2015;31:557-74.

8. Sonar S, Lal G. Role of tumor necrosis factor superfamily in neuroinflammation and autoimmunity. Front Immunol 2015;6:364.

9. Dowlati Y, Herrmann N, Swardfager W, et al. A meta-analysis of cytokines in major depression. Biol Psychiatry 2010;67:446-57.

10. Sharaf K, Ihler F, Bertlich M, Reichel C, Berghaus A, Canis M. Tumor Necrosis Factor-induced decrease of cochlear blood flow can be reversed by Etanercept or JTE-013. Otol Neurotol 2016;37: e203-8.
11. Ihler F, Pelz S, Coors M, Matthias C, Canis M. Application of a TNF-alpha-inhibitor into the scala tympany after cochlear electrode insertion trauma in guinea pigs: preliminary audiologic results. Int $J$ Audiol 2014;53:810-6.

12. Chen G, Goeddel DV. TNF-R1 signaling: a beautiful pathway. Science 2002;296:1634-5.

13. Suckfuell M, Canis M, Strieth S, Scherer H, Haisch A. Intratympanic treatment of acute acoustic trauma with a cell-permeable JNK ligand: a prospective randomized phase I/II study. Acta Otolaryngol 2007;127:938-42.

14. Ihler F, Sharaf K, Bertlich M, et al. Etanercept prevents decrease of cochlear blood flow dose-dependently caused by tumor necrosis factor alpha. Ann Otol Rhinol Laryngol 2013;122:468-73.

15. O'Connor P, Comi G, Montalban X, et al. Oral fingolimod (FTY720) in multiple sclerosis: two-year results of a phase II extension study. Neurology 2009;72:73-9.

16. Kappos L, Antel J, Comi G, et al. Oral fingolimod (FTY720) for relapsing multiple sclerosis. $N$ Engl J Med 2006;355:1124-40.

17. Brinkmann V, Davis MD, Heise CE, et al. The immune modulator FTY720 targets sphingosine 1-phosphate receptors. J Biol Chem 2002;277:21453-7.

18. Matloubian M, Lo CG, Cinamon G, et al. Lymphocyte egress from thymus and peripheral lymphoid organs is dependent on S1P receptor 1. Nature 2004;427:355-60.

19. Ihler F, Bertlich M, Sharaf K, Strieth S, Strupp M, Canis M. Betahistine exerts a dose-dependent effect on cochlear stria vascularis blood flow in guinea pigs in vivo. PLoS One 2012; 7:e39086.

20. Bertlich M, Ihler F, Freytag S, Weiss BG, Strupp M, Canis M. Histaminergic H3-heteroreceptors as a potential mediator of betahistine-induced increase in cochlear blood flow. Audiol Neurotol 2015;20:283-93.

21. Bertlich M, Ihler F, Sharaf K, Weiss BG, Strupp M, Canis M. Betahistine metabolites, aminoethylpyridine, and hydroxyethylpyridine increase cochlear blood flow in guinea pigs in vivo. Int $J$ Audiol 2014;53:753-9.

22. Zeintl H, Sack FU, Intaglietta M, Messmer K. Computer assisted leukocyte adhesion measurement in intravital microscopy. Int $J$ Microcirc Clin Exp 1989;8:293-302.

23. Baker M, Wayland $\mathrm{H}$. On-line volume flow rate and velocity profile measurement for blood in microvessels. Microvasc Res 1974;7: 131-43.

24. Du J, Zeng C, Li Q, et al. LPS and TNF-alpha induce expression of sphingosine-1-phosphate receptor-2 in human microvascular endothelial cells. Pathol Res Pract 2012;208:82-8.

25. Zhang G, Yang L, Kim GS, et al. Critical role of sphingosine-1phosphate receptor 2 (S1PR2) in acute vascular inflammation. Blood 2013;122:443-55.

26. Paugh SW, Cassidy MP, He H, et al. Sphingosine and its analog, the immunosuppressant 2-amino-2-(2-[4-octylphenyl] ethyl)-1,3-propanediol, interact with the CB1 cannabinoid receptor. Mol Pharmacol 2006;70:41-50.

27. Kianian M, Kelly MEM, Zhou J, et al. Cannabinoid receptor 1 inhibition improves the intestinal microcirculation in experimental endotoxemia. Clin Hemorheol Microcirc 2014;58:333-42.

28. van der Poll T, Coyle SM, Levi M, et al. Effect of a recombinant dimeric tumor necrosis factor receptor on inflammatory responses to intravenous endotoxin in normal humans. Blood 1997;89: $3727-34$.

29. Mutschler D, Wikstrom G, Lind L, Larsson A, Lagrange A, Eriksson M. Etanercept reduces late endotoxin-induced pulmonary hypertension in the pig. J Interferon Cytokine Res 2006;26: 661-7.

30. Coleman JKM, Littlesunday C, Jackson R, Meyer T. AM-111 protects against permanent hearing loss from impulse noise trauma. Hear Res 2007;226:70-8.

31. La Mantia L, Tramacere I, Firwana B, Pacchetti I, Palumbo R, Filippini G. Fingolimod for relapsing-remitting multiple sclerosis. Cochrane database Syst Rev 2016;4:CD009371. 